\title{
NONDIFFERENTIABILITY OF RETRACTIONS OF $C^{n}$ TO SUBVARIETIES
}

\author{
M. GILMARTIN
}

Theorem 5.2 of [1] says that if $x$ is a singular point of a complex subvariety $V$ of an open subset of $C^{n}$, then for no open neighborhood $N$ of $x$ in $C^{n}$ is there a holomorphic retraction of $N$ to $V \cap N$. It will be proved here that there isn't even a real- $C^{1}$ retraction.

LEMMA. If a complex subvariety $V$ of an open subset $U$ of $C^{n}$ is a real-C $C^{1}$ submanifold, it is a complex submanifold.

Proof. The set $R(V)$ of points of $V$ having open neighborhoods in $V$ which are complex submanifolds of open subsets of $C^{n}$ is dense in $V$. For $y \in V$ let $V_{y}$ denote the tangent space at $y$ to the real- $C^{1}$ submanifold $V$ of $U$. $V_{y}$ is a real-linear subspace of $C^{n}$ for all $y \in V$. Let $A$ be the set of all $y \in V$ such that $V_{y}$ is a complex-linear subspace of $C^{n}$. Then $A$ is a relatively closed subset of $V$. Also, since $R(V) \subseteq A$, $A$ is dense in $V$. Hence $A=V . V_{y}$ is a complex-linear subspace of $C^{n}$ for all $y \in V$. So $V$ is a complex submanifold of $U$.

TheOREM. Let $V$ be a complex subvariety of an open set $U \subseteq C^{n}$. Let $x \in V$. Suppose there exist an open neighborhood $U_{1}$ of $x$ in $U$ and $a$ real- $C^{1}$ retraction $F$ of $U_{1}$ to $V \cap U_{1}$. Then $x \in R(V)$.

Proof. Choose a real- $C^{1}$ submanifold $S$ of an open neighborhood $U_{2}$ of $x$ in $U_{1}$ such that $S \supseteq V \cap U_{2}$ and the real dimension $s$ of $S$ is minimal for this property. If $s=0$ then $x$ is an isolated point of $V$, and hence in $R(V)$. So suppose $s>0$. If $f$ is a real- $C^{1}$ function on $S$ such that $(d f)_{x} \neq 0$, then the set $S_{1}$ of zeros of $f$ is a submanifold of real dimension $s-1$ in some neighborhood of $x$ in $S$, so by the minimality of the dimension of $S, S_{1}$ contains no neighborhood of $x$ in $V$, so $f$ vanishes on no neighborhood of $x$ in $V$. In other words, if $f$ is a real- $C^{1}$ function on $S$ vanishing on $V \cap U_{2}$, then $(d f)_{x}=0$. If $f$ and $g$ are real$C^{1}$ functions on $S$ which coincide on $V \cap U_{2}$, then $(d f)_{x}=(d g)_{x}$. Since $F$ equals the identity on $V \cap U_{2},(d(F \mid S))_{x}=(d I)_{x}$, where $I$ is the identity on $S$. Thus $(d(F \mid S))_{x}$ has rank $s$. By the inverse function theorem $F(S) \subseteq V$ is a neighborhood of $x$ in $S$. There is an open neighborhood $U_{3}$ of $x$ in $U_{2}$ such that $V \cap U_{3}=S \cap U_{3}$. By the lemma, $V \cap U_{3}$ is a complex submanifold of $U_{3} . x \in R(V)$.

Received by the editors June 4, 1964 . 


\section{REFERENCE}

1. H. Rossi, Vector fields on analytic spaces, Ann. of Math. 78 (1963), 455-467.

Princeton University

\section{ON PIECEWISE LINEAR IMMERSIONS}

\section{MORRIS W. HIRSCH}

The purpose of this note is to prove an existence theorem for immersions of piecewise linear manifolds in Euclidean space. A more comprehensive theory of piecewise linear immersions has been worked out by Haefliger and Poenaru [1].

All maps, manifolds, microbundles, etc. are piecewise linear unless the contrary is explicitly indicated.

Let $M$ be a manifold without boundary, of dimension $n$. Denote the tangent microbundle of $M$ by $\tau_{M}$, and the trivial microbundle over $M$ of (fibre) dimension $k$ by $\epsilon^{k}$. Let

$$
\nu: M \stackrel{i}{\rightarrow} E \stackrel{j}{\rightarrow} M
$$

be a microbundle of dimension $k$ such that $E$ is a manifold. An immersion of $M$ in $R^{n+k}$ is a locally one-one map $f: M \rightarrow R^{n+k}$.

I say $f$ has a normal bundle of type $\nu$ if there is an immersion $g: E \rightarrow R^{n+k}$ such that $g i=f$. (It is unknown whether $f$ necessarily has a normal bundle, or whether all normal bundles of $f$ are of the same type.)

The converse of the following theorem is trivial.

Theorem. Assume that if $k=0$, then $M$ has no compact component. There exists an immersion of $M$ in $R^{n+k}$ having a normal bundle of type $\nu$ if there exists an isomorphism

$$
\phi: \tau_{m} \oplus \nu \rightarrow \epsilon^{n+k}
$$

Proof. We may assume that $i(M)$ is a deformation retract of the total space $E$ of $\nu$. By Milnor [3], $\tau_{E} \mid i(M)$ is isomorphic to $\tau_{M} \oplus \nu$; it follows from the existence of $\phi$ that $\tau_{E}$ is trivial. According to [3]

Received by the editors July 29, 1964. 